\title{
Solar ALMA predictions: tutorial
}

\author{
R. J. Rutten \\ Lingezicht Astrophysics, 't Oosteneind 9, 4158 CA Deil, The Netherlands \\ National Astronomical Observatory of Japan, 2-21-1 Osawa, Mitaka, Tokyo 181-8588, Japan \\ email: R.J.Rutten@uu.nl
}

\begin{abstract}
I have proposed that long $\mathrm{H} \alpha$ fibrils are caused by heating events of which the tracks are afterwards outlined by contrails of cooling gas with extraordinary $\mathrm{H} \alpha$ opacity and yet larger opacity at the ALMA wavelengths. Here I detail the radiative transfer background.
\end{abstract}

\section{Introduction}

Recently I proposed that many long $\mathrm{H} \alpha$ fibrils, i.e., the slender $\mathrm{H} \alpha$ features that extend from plage and network far out over adjacent quiet internetwork areas wherever the Sun shows some activity, are contrails (Rutten 2017, henceforth Pub 1). Airplane contrails on our sky are water recondensation features tracking sudden heating in jet engines. $\mathrm{H} \alpha$ contrails on the solar disk are hydrogen recombination features tracking sudden heating in jet-like heating events. A striking example with precursor heating to very high temperature was detailed by Rutten \& Rouppe van der Voort (2017).

Observationally, I based this proposal not only on the latter example but also on observed paucity of internetwork shock scenes in $\mathrm{H} \alpha$, large disalikeness between $\mathrm{H} \alpha$ and Ly $\alpha$ images, and fibril incongruity between $\mathrm{H} \alpha$ and Ca II $8542 \AA$.

Theoretically, I based this proposal on appreciating how Ly $\alpha$ controls hydrogen populations in neutral-hydrogen gas and so produces non-equilibrium (non-E) ionizationrecombination balancing in cooling hydrogen gas.

A corollary is that such $\mathrm{H} \alpha$ contrails are as opaque or much more opaque at the ALMA wavelengths. Section 5 of Pub 1 gives a dozen explicit predictions of what ALMA may therefore see and not see on the Sun. In a nutshell, I predict that internetwork areas are covered by opaque canopies wherever $\mathrm{H} \alpha$ shows fibrils, but as umbrellas rather than fibrilar canopies, i.e., opaque blankets with less lateral contrast. These will obscure not only activity features underneath such as Ellerman bursts (Ellerman 1917; review by Rutten et al. 2013), but also quiet-Sun features as the predicted shock scenes in e.g., Fig. 4 of Wedemeyer (2016) - unless we sink in a Maunder minimum.

I presented the above at the symposium. Here I detail radiative transfer background that was only summarized in Sect. 2.1 of Pub 1 by linking to a selection of my solar spectrum example displays as a shortcut to avoid inclusion of more figures. I show these figures here and explain them with references to my ADS-available course notes (Rutten 2003, henceforth RTSA), which are largely a didactic rendering of material covered already by Mihalas (1970). Although optically thick radiative transfer is long-standing textbook material, it remains intransparent. I therefore use graphical exposition here in the order LTE - NLTE - non-E. I also treat Rydberg lines (skipped in Pub 1). $\dagger$

$\dagger$ Links to cited figures and equations open the pertinent page in a browser depending on your pdf viewer, its permission setup, and your publisher access. The name-year clickers open the ADS abstract page. Acronyms may show their meaning at mouse-over (not in Preview). 


\section{Hydrogen in Saha-Boltzmann equilibrium}

ALMA as thermometer. ALMA is often advertised as a linear thermometer of the solar atmosphere, quoting "LTE formation of solar mm continua". LTE holds strictly only for the source function of free-free (ff) processes since these are collisional and the Maxwell distribution is valid in chromospheric conditions. However, $S \approx B$ is a good approximation for $\mathrm{mm}$ bound-free (bf) continua which do not suffer the tremendous scattering with $S \approx J$ that characterizes all strong solar lines and also all bf ultraviolet continua (examples below). The assumption is wrong for Thomson and Rayleigh scattering, but their contributions are negligible in chromospheric conditions at mm wavelengths, also unlike the optical and ultraviolet.

Since solar mm radiation obeys the Rayleigh-Jeans approximation, ALMA-measured brightness temperature from optically thick features therefore indeed represents electron temperature when absolute intensity calibration is achieved. The signal then represents $T\left(\tau_{\mu}=1\right)$, i.e., the temperature at the representative Eddington-Barbier location defined by the summed extinction along the line of sight. Optically thin features show (apart from their background) $T_{\mathrm{b}}=\tau T$, a fraction of the temperature scaled by the summed extinction along the beam. The question therefore is one of extinction - is it LTE, NLTE, non-E, and how thick does it make a feature of interest at $\mathrm{mm}$ wavelengths? I start with LTE and then treat NLTE and non-E which require non-local multi-level scrutiny of hydrogen population processes.

Saha-Boltzmann opacities. The definition of LTE is local validity of Saha and Boltzmann (SB) partitioning for the populations of all pertinent particle levels and stages (Sect. 2.5 on page 28 of RTSA). The extinction coefficient for any transition can then be evaluated from the local temperature, electron density, and elemental abundance without knowledge of impinging radiation fields, even if these do not correspond to the local temperature. Iterative solution is required because the electron density enters reciprocally in the Saha population ratio between successive ionization stages and depends on the degree of ionization of hydrogen, helium, and abundant metals and on the degree of pertinent molecular binding, in particular of $\mathrm{H}$ into $\mathrm{H}_{2}$ (Sect. 7.2.2 on page 143 of RTSA; more detail in Sects. 3.1 and 3.2 of Mihalas 1970).

The lower panels of Fig. 1 show resulting densities for gas of solar composition. In the small plateau near $4000 \mathrm{~K}$ the free electrons with $N_{\mathrm{e}} \approx 10^{-4} N_{\mathrm{H}}$ come from abundant low-ionization metals $(\mathrm{Mg}, \mathrm{Fe}, \mathrm{Si}, \mathrm{Al})$; these govern photospheric $\mathrm{H}^{-}$extinction. At right virtually all free electrons come from hydrogen.

The SB extinction curves in the upper panels of Fig. 1 follow the hydrogen ionization. The decay slope for $\operatorname{Ly} \alpha$ is the same as for $N_{\mathrm{HI}}$, but the decay of $\mathrm{H} \alpha$ is largely compensated by the increasing Boltzmann ratio (for $\mathrm{SB} \mathrm{H} \alpha$ even exceeds Ly $\alpha$ above $40000 \mathrm{~K}$ from $\left.g_{2} / g_{1}=8\right)$. The Boltzmann factor makes the initial rise for $\mathrm{H} \alpha$ exceedingly steep, spanning over 10 dex (orders of magnitude). $\mathrm{H} \alpha$ extinction is nearly negligible below $5000 \mathrm{~K}$, which explains why the CaII H \& K lines show much larger dips in the photospheric spectrum, but above $10000 \mathrm{~K}$ the SB extinction of $\mathrm{H} \alpha$ becomes much larger than that of $\mathrm{Mg}$ II $\mathrm{k}$ and at high density even comparable to that of the C II and Si IV resonance lines (Fig. 5 of Rutten 2016b).

In Fig. $1 \mathrm{H}^{-} \mathrm{ff}$ extinction is the main continuum agent below $5000 \mathrm{~K}$ while $\mathrm{HIff}$ extinction, sharing the steep Boltzmann increase, wins at higher temperature. Therefore, across the parameter domain of Fig. 1 the ff-process $S=B$ equality indeed holds.

Comparison of these curves with the horizontal line at $y=-7$ shows that in $\mathrm{SB}$ conditions a feature of $100 \mathrm{~km}$ thickness and temperature above $6000 \mathrm{~K}$ is optically thick 

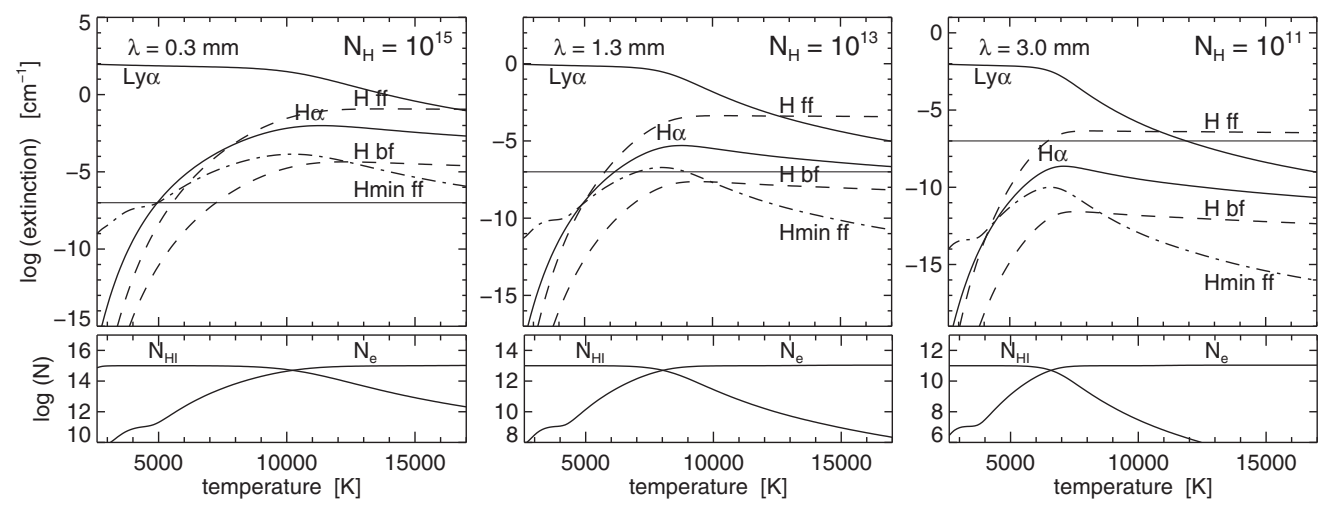

Figure 1. Saha-Boltzmann line extinction coefficient against temperature at the centers of Ly $\alpha$ and $\mathrm{H} \alpha$ (solid) and continuous extinction coefficient of the $\mathrm{HI}$ free-free and bound-free contributions (dashed) and the $\mathrm{H}^{-}$free-free contribution (dot-dashed) at three ALMA wavelengths (from left to right $0.35,1.3$ and $3.0 \mathrm{~mm}$ ), for gas of solar composition with different total hydrogen densities (from left to right $N_{\mathrm{H}}=10^{15}, 10^{13}$ and $10^{11} \mathrm{~cm}^{-3}$, corresponding to the radiation escape heights at these wavelengths at the bottom, middle and top of the ALC7 chromosphere). The horizontal line at $y=-7$ shows at which extinction a $100 \mathrm{~km}$ thick feature becomes optically thick. The small lower panels show the competing neutral hydrogen and electron densities $\left(\right.$ part $\mathrm{cm}^{-3}$ ), at the same logarithmic unit (dex) size as the extinction scales to enable slope comparisons. The $y$ scales shift between columns. Selection from Fig. 1 of Pub 1.

to extremely thick in $\mathrm{H} \alpha$ and $\mathrm{H}$ Iff in the first column, still thick in the second, thin in $\mathrm{H} \alpha$ but still thickish in H Iff in the third. The difference H Iff- $\mathrm{H} \alpha$ increases with wavelength because $\alpha_{\nu}^{\mathrm{ff}} \sim \lambda^{2} N_{\mathrm{e}} N_{\text {ion }} T^{-3 / 2}$ (Eq. 2.79 on page 27 of RTSA). Hence, if solar features have $\mathrm{SB}$ opacities then their visibility in $\mathrm{H} \alpha$ implies equal or better visibility at the ALMA wavelengths, more so at higher temperature and longer wavelength.

\section{Hydrogen in static NLTE solar model atmospheres}

Standard models. I now turn to hydrogen behavior in NLTE, using the unrealistic simplification of static plane-parallel modeling because hypothetical idealized atmospheres of this type have the didactic virtue of producing fully understandable spectra†. See Rutten (2002) for a review of such modeling.

Figure 2 shows two recent contenders. I prefer ALC7 because its chromospheric extent is based on turbulent pressure constrained by observed non-thermal line broadening, similarly to the predecessor VALIII models of Vernazza et al. (1981) and FAL models of Fontenla et al. (1993), whereas the extent of FCHHT-B (without the A of Avrett) is based on ad-hoc gravity modification in addition to this turbulent pressure. The FCHHT$\mathrm{B}$ model has a higher-located and appreciably cooler temperature minimum, introduced to reproduce observed deep CO lines that in reality are due to non-static non-E cooling clouds (Uitenbroek 2000; Asensio Ramos et al. 2003). I add this model here because its steep temperature rise to its chromosphere produces didactically valuable Ly $\alpha$ back-radiation discussed below. The near-isothermal chromospheres of both models are effectively constrained by acoustic shocks in internetwork areas and represent their temperatures, densities, and degree of hydrogen ionization rather than quiet-Sun averages of these quantities (Carlsson \& Stein 1995).

$\dagger$ I regard 1D models as computationally existing didactically superb non-solar-like planeparallel stars with remarkably solar-like but misleading spectra (page 189 of RTSA). 

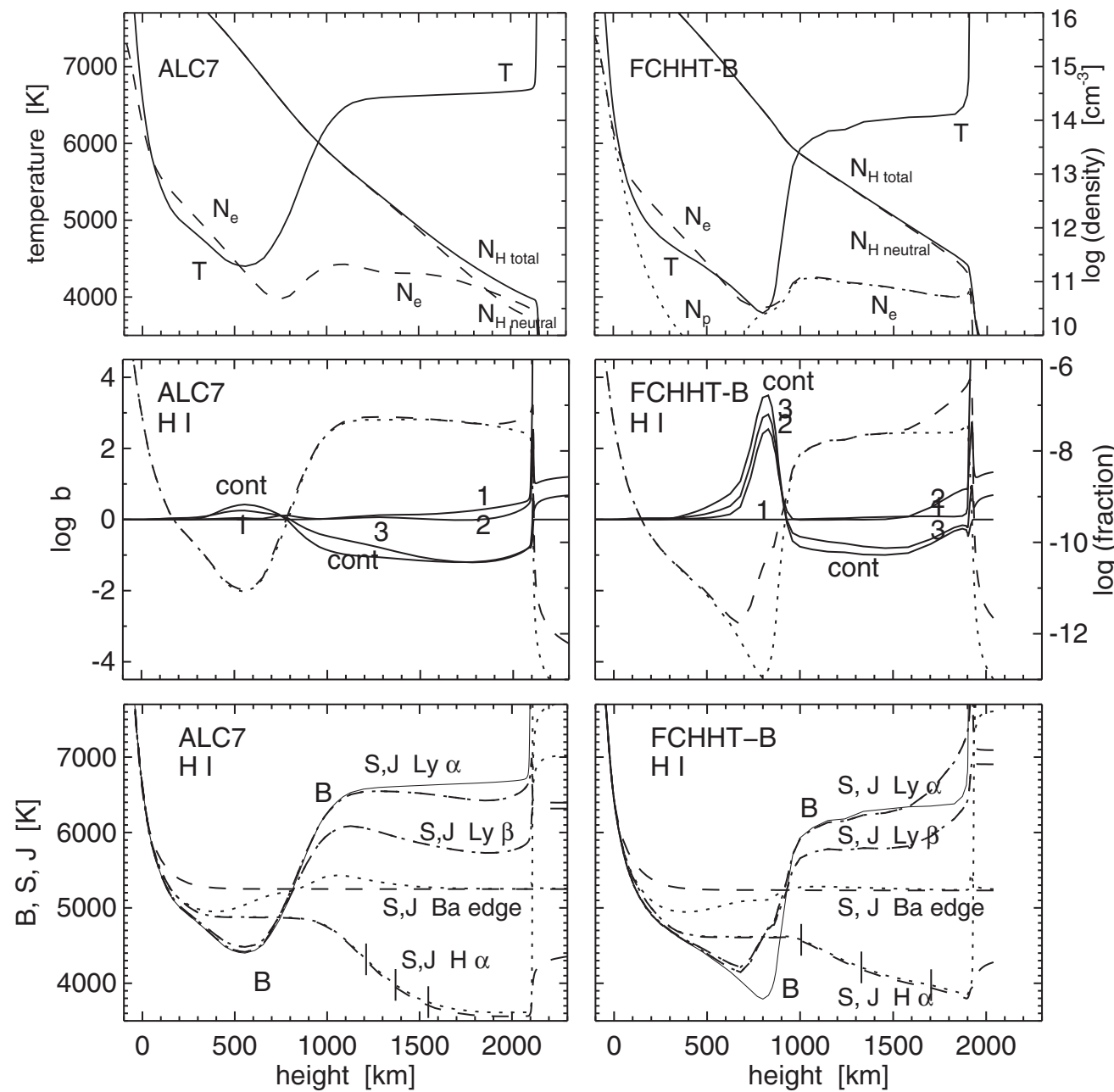

Figure 2. Hydrogen in static plane-parallel atmospheres. Left: ALC7 of Avrett \& Loeser (2008). Right: FCHHT-B of Fontenla et al. (2009). Top row: model properties against height. Density scale at right. Center row: NLTE departure coefficients for hydrogen levels $1-3$ and the continuum. The dashed curves show the fractional population $n_{2} / N_{\mathrm{H}}$ of the $n=2$ hydrogen level in NLTE, the dotted curves in LTE, with scale at right. Bottom row: $B, S$ and $J$ for hydrogen features, plotted as representative temperatures. The ticks on the $S$ curves for $\mathrm{H} \alpha$ are for $\tau=3,1,0.3$. The Balmer edge curves are $100 \AA$ averages shortward of $3646 \AA$ including lines.

In both models the photospheric temperature gradient is close to radiative equilibrium. The electron density follows $N_{\mathrm{H}}$ at the $10^{-4}$ offset governed by low-ionization metals until hydrogen ionizes. It then remains fairly constant throughout the model chromospheres, and so is therefore the collisional destruction probability $\varepsilon$ (Sect. 3.4 on page 64 of RTSA) which scales primarily with $N_{\mathrm{e}}$ (Eq. 3.33 on page 50 of RTSA). Since the temperature is also fairly constant these model chromospheres are similar to the classic isothermal constant- $\varepsilon$ atmosphere for which the theory of scattering line formation was developed half a century ago, in particular in Avrett (1965) (Sect. 4.3 on page $92 \mathrm{ff}$ of RTSA). 
NLTE populations. The center panels of Fig. 2 show NLTE population departure coefficients $\dagger=n / n^{\text {LTE }}$ for the first three levels and the continuum state of hydrogen (free protons). Below the transition region the ground level has $b_{1} \approx 1$, as expected since where hydrogen is mostly neutral almost all hydrogen particles sit in that level. The second level also shows near-SB population, except in the temperature minimum and the onset to the transition region. The third level has $b_{3}$ dropping towards the continuum offset $b_{\text {cont }} \approx 0.1$ across both chromospheres. The FCHHT-B model has high $b$ peaks for the higher levels in its temperature minimum. Except for $b_{1}$ these behaviors have to do with the $\operatorname{Ly} \alpha, \mathrm{H} \alpha$ and Balmer-continuum source functions. These are the main topic here.

Meaning of NLTE. NLTE means that LTE cannot be assumed. Usually it means that statistical equilibrium (SE) is assumed. Often, it is taken to mean only what I call source function NLTE: $S \neq B$ - but often for photospheric lines opacity NLTE is more important, e.g., for Fe I lines (Rutten 1988 and below).

NLTE is primarily about scattering, i.e., effects of impinging radiation brought from elsewhere by scattering. This non-local influence can indeed affect line source functions, in the Wien approximation (valid for Lyman and Balmer transitions) measured as $S^{l} \approx$ $\left(b_{u} / b_{l}\right) B$, but also line extinction coefficients, in the Wien approximation measured as $\alpha^{l} \approx b_{l} \alpha_{\text {LTE }}^{l}$ (Sect. 2.6.2 on page 33 of RTSA). Source function NLTE $\left(b_{u} / b_{l} \neq 1\right)$ is usually due to bb scattering, opacity NLTE $\left(b_{l} \neq 1\right)$ to bf scattering. I showcase both.

Bound-bound scattering. The bb line source function can be decomposed into distinct contributions as

$$
S^{l}=(1-\varepsilon-\eta) J+\varepsilon B+\eta S^{\mathrm{D}},
$$

weightedly summing different sources for producing new line photons: scattering from the local photon reservoir (angle-averaged intensity $J$ ), creation by collisional excitation using thermal energy described by $B$, and detour production via lower-to-upper paths along other levels and stages, with $S^{\mathrm{D}}$ a formal combined source function for all such paths which may include ionization plus recombination (teaching display "all bb pairs").

This is a fundamental equation but "not yet" in RTSA - I believe it has been published only by me so far in this form using $\varepsilon$ (fraction of photoexcitations followed by direct collisional upper-to-lower deexcitation) and $\eta$ (fraction of photoexcitations followed by detour upper-to-lower paths). The classic literature used instead the ratios $\varepsilon^{\prime}$ and $\eta^{\prime}$ of such extinctions to the contribution by two-level scattering following Thomas (1957) (e.g., Eq. 8.5 on page 181 of Jefferies 1968).

Figure 3 shows the formation of $\mathrm{NaI}_{1}$ in $\mathrm{ALC} 7$ because this line presents a clean example of bb resonance scattering. The detour terms are negligible for this line, and also the effects of coherent scattering (partial redistribution, PRD) can be ignored (Uitenbroek $\&$ Bruls 1992). However, its $\varepsilon \approx 10^{-3}$ in the chromosphere means that resonance scattering dominates there. The line source function (at line center $\approx$ total source function because the contribution by continuous extinction processes is negligible, Eq. 2.23 on page 13 of RTSA) follows the scattering decline illustrated by Avrett (1965) to the small value at the escape surface given by the " $\sqrt{\varepsilon}$ " law with $I \approx S(\tau=1) \approx S(\tau=0)=\sqrt{\varepsilon} S$ (Eq. 4.81 on page 97 of RTSA). Correspondingly, the thermalization depth is large: $\Lambda \approx 1 / \varepsilon$ in optical depth units for the Doppler core and complete redistribution (CRD) (Eq. 4.106 on page 110 of RTSA). This is roughly the depth at which the radiation does not yet

$\dagger$ Beware: these are Zwaan coefficients, not Menzel coefficients used by E.H. Avrett and J. Fontenla. See warning on page 36 of RTSA regarding misinterpretation, as indeed done by Fontenla et al. (2009) and diagnosed by Rutten \& Uitenbroek (2012). 

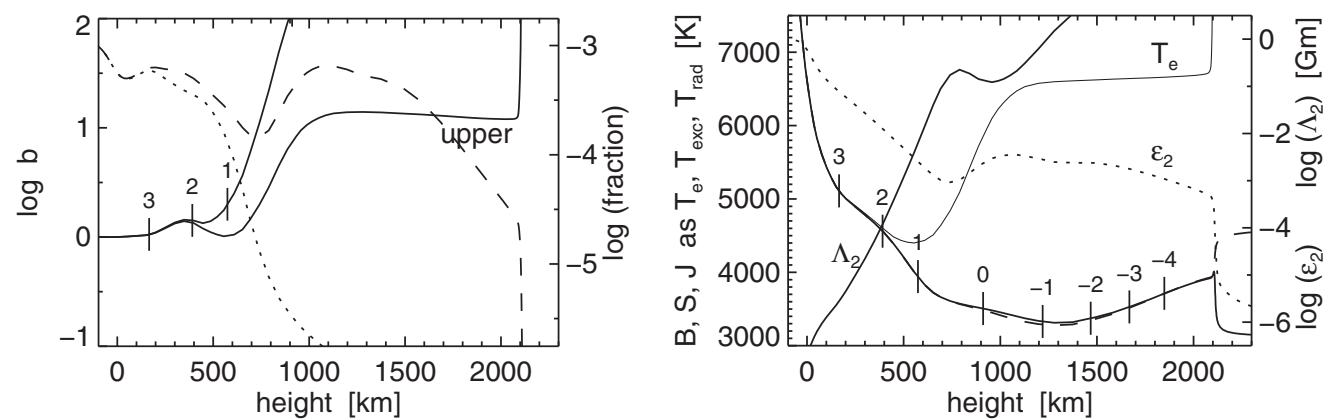

Figure 3. Formation of $\mathrm{NaI} \mathrm{D}_{1}$ in the ALC7 atmosphere. Left: departure coefficients $b_{l}$ and $b_{u}$ (solid) and fractional lower-level population in NLTE (dashed) and LTE (dotted) with scale at right. Right: $B, S$ (solid) and $J$ (dashed) represented as formal temperatures (electron, excitation, radiation defined on page $37 \mathrm{ff}$ of RTSA). Dotted: collisional destruction probability $\varepsilon$ in two-level approximation (ignoring detours). Scale to the right. Dot-dashed: thermalization length for the Doppler core in Gigameter, same scale. The label $\Lambda_{2}$ is placed near the line-core thermalization height. The numbered ticks show values of $\log \tau$ at $\operatorname{line}$ center; $\log \tau=0$ represents the characteristic Eddington-Barbier sampling height for vertical viewing.

sense the presence of a surface where it can leak out and therefore remains controlled by the local temperature (Avrett's Fig. 4.13 on page 109 of RTSA).

Their scattering makes the Na I D lines the darkest solar lines in the optical spectrum. Figure 3 shows that the $\mathrm{NaI} \mathrm{D}_{1}$ core is chromospheric in ALC7 in the sense that the last scattering encoding Dopplershift and Zeeman signature takes place around $900 \mathrm{~km}$, but the core photons that escape there were thermally created near $200 \mathrm{~km}$ in the low ALC7 photosphere. Its core intensity does not sense the presence of the ALC7 chromosphere but rather responds to disturbances in the low photosphere.

The NaI $D_{1} S / B$ scattering ratio translates into a $b_{u} / b_{l}$ ratio at left. Their divergences would be the same in logarithmic units if $B$ and $S$ were plotted directly, but I use representative temperatures to remove nonlinear Wien sensitivities for comparisons with other wavelengths.

The $\mathrm{NaI} \mathrm{D}_{1} b_{l}$ curve shows a steep increase in the chromosphere because there $\mathrm{Na}$ ionization does not follow temperature (below). This increase offsets the steep decline that the ground level population would have according to the Saha equation (dotted).

Bound-free scattering. In principle bf scattering is the same as bb scattering: the equation description can be unified (Sect. 3.2.3 on page 48 of RTSA). However, there are qualitative differences in important properties:

(a) there is no coherency (PRD) in bf scattering because each recombination employs a fresh electron; complete frequency redistribution (CRD) occurs over the full bf edge requiring averaging integrals over its extent (Eq. 3.109 on page 73 of RTSA);

(b) the $\Lambda$ operator which produces angle-averaged radiation intensity $J$ from the total source function (Sect. 4.1 .3 on page $81 \mathrm{ff}$ of RTSA) gives $\Lambda(S) \approx S$ when $S \sim 1+1.5 \tau$, but produces $J>S$ for steeper $S(\tau)$ (Kourganoff's Fig. 4.4 on page 83 of RTSA);

(c) the $T(h)$ decay is set by radiative equilibrium $S \approx J$ in the optical part of the spectrum where the emergent flux peaks, there forcing $S \sim 1+1.5 \tau$ (Sect. 7.3.2 on page $153 \mathrm{ff}$ of RTSA). In the ultraviolet Wien non-linearity produces steepening of the Planck function for this given gradient (Fig. 4.9 on page 101 of RTSA);

(d) a bf edge has similar total (spectrum-integrated) extinction as a bb resonance line, but because it is much wider its monochromatic extinction coefficient is much smaller. This means that the typical $B\left(\tau_{\lambda}\right)$ gradient remains steeper, while in resonance lines it 

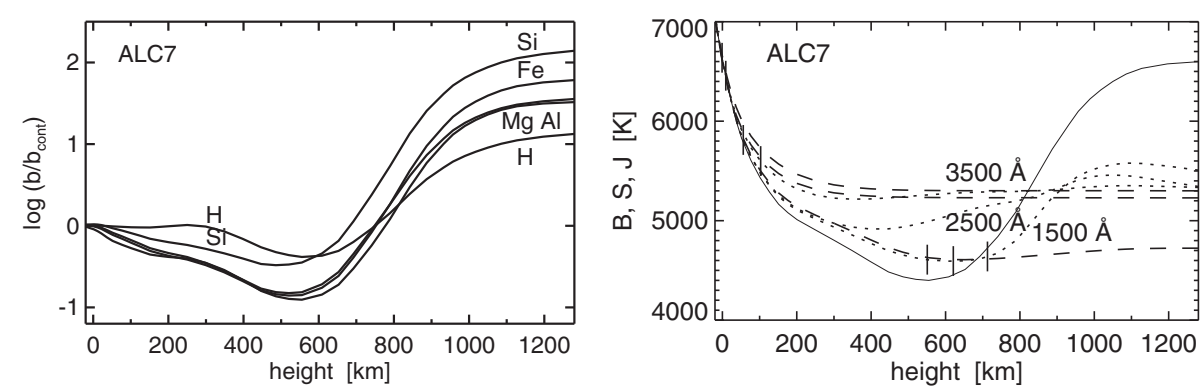

Figure 4. Left: departure coefficient ratios $b_{2} / b_{\text {cont }}$ for HI and $b_{1} / b_{\text {cont }}$ for Mg I, FeI, SiI, Al I. Right: $B$ (solid), $S$ (dotted) and $J$ (dashed) as representative temperatures at 3500,2500 , $1500 \AA$. $S$ and $J$ are $100 \AA$ averages including lines. The ticks are at $\tau=3,1,0.3$.

gets so shallow from the additional line extinction that it approaches the isothermal case obeying the $\sqrt{\varepsilon}$ law (Fig. 4.10 on page 103 of RTSA);

(e) the solar continuum opacity does not only have its well-known minimum at the $\mathrm{H}^{-}$bf threshold wavelength at 1.6 micron, but also a (nearly as deep) minimum at $4000 \AA$ because only shortward of $4000 \AA$ the metal bf edges become more important than $\mathrm{H}^{-}$bf extinction(Vitense's Fig. 8.6 on page 179 of RTSA). Therefore, near-ultraviolet radiation escapes very deep and there the $T(h)$ gradient is steep.

The combined result of these bf properties is that ultraviolet continua escape by scattering out from the deep photospheret, with $B$ gradients steeper than the resulting outward $S \approx J$ scattering declines. Figure 4 illustrates this for the Balmer continuum and the electron-donor continua in the ultraviolet, whose edges (wavelengths specified in Table 9 on page 665 of Vernazza et al. 1981 and in Table 8.1 on page 176 of RTSA) together produce the increasing extinction that makes the solar ultraviolet escape at larger height for shorter wavelength. The $b$ ratios at left show a dip in the temperature minimum followed by a steep increase that both result from following $J$ rather than $B$, illustrated by the $S \approx J-B$ offsets at three ultraviolet sample wavelengths at right.

The electron donors have $b_{\text {cont }} \approx 1$ because they are largely ionized; their ratio curves at left represent under- and overpopulation of their ground levels. The deep initial dip for FeI, MgI and Al I implies that all photospheric lines arising from their low-lying levels have increasing and significant extinction depletion across the photosphere. For examples see my display $10=\mathrm{Mg}$ I 4571 from ALC7 and display $11=$ Fe I 6301.5 from ALC7. Such depletion occurs generally for minority-species lines.

The exceptions are alkali lines which due to low ionization energy suffer strong photon suction: population pull-down from the continuum reservoir by photon losses in strong lines, see Bruls et al. (1992) or Sect. 10.1 on page $213 \mathrm{ff}$ of RTSA. It produces the initial hump rather than dip at $400 \mathrm{~km}$ in the $\mathrm{NaI} \mathrm{D}_{1} b_{l}$ curve in Fig. 3.

The scattering nature of ultraviolet continua has been illustrated best for the VALIIIC model (at 50 citations/year the most famous plane-parallel star) in the wonderful elevenpage Fig. 36 on page $666 \mathrm{ff}$ of Vernazza et al. (1981), with a selection copied on page $184 \mathrm{ff}$ of RTSA.

$\dagger$ When the metal edges vanish through ionization only the Balmer continuum remains with yet deeper escape. This happens in kilogauss magnetic concentrations ("fluxtubes") from evacuation by magnetic pressure producing low gas density (Spruit 1977). Their brightening in 1600 and $1700 \AA$ images from TRACE and AIA is not from photospheric heating but from hole-in-the-surface radiation just as "line-gap" brightening of FeI cores (Vitas et al. 2009). The larger brightening of Ellerman bursts at 1600 and $1700 \AA$ is also from photospheric metal ionization, but through intrinsic heating at increased density (Rutten 2016b). 

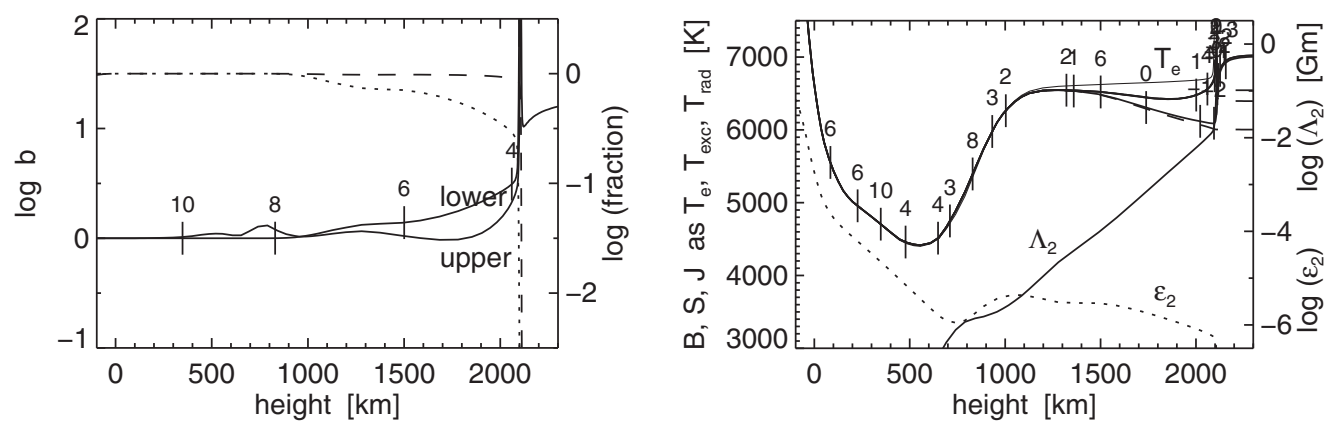

Figure 5. Ly $\alpha$ in the ALC7 chromosphere, in the format of Fig. 3. Because Ly $\alpha$ is a PRD line there are three line source functions shown at right, for line center, the profile peaks, and the profile dips, each with (confusingly mixed) $\log \tau$ ticks but only the line-center ones at left.

Line haze modeling. In the violet and ultraviolet myriads of neutral and once-ionized metal lines together constitute a quasi-continuous "line haze" (Greve \& Zwaan 1980) which lowers ultraviolet $J>B$ excesses. Quantification is non-trivial because these lines are all scatterers themselves, with much multi-level interlocking between multiplets and multiplet members. Their effect was still underestimated in the VALIII model; all Avrett and Fontenla models since Maltby et al. (1986) therefore share a less steep photospheric temperature decline closer to classic LTE and radiative-equilibrium models (Fig. 7.3 on page 149 of RTSA).

In Figs. 2-6 the line-haze lines are accounted for with an individual two-level scattering approximation (only the $\varepsilon$ terms in Eq. 3.1) in the RH code of Uitenbroek (2001) used for their production (with IDL plotting programs available on my website). I used an RH setup sampling 340000 ultraviolet and optical lines from the list of Kurucz (2009) at $20 \mathrm{~m} \AA$ wavelength spacing. This approach overestimates line depths for many weaker lines (all those connected to stronger ones via their upper level or via levels close to their upper level), in particular for most optical lines from the photosphere, by overestimating their scattering and resulting $S<B$ departure. However, in the ultraviolet where the line haze is most important such scattering is a better assumption than LTE $S=B$ which gives non-observed central reversals for all lines with $\tau \approx 1$ in the model chromosphere. In the construction of the ALC7 model all Kurucz lines were set to transit from $S=B$ in the photosphere to $S=J$ in the chromosphere with a gradual change-over factor comparable to $\varepsilon$ in Eq. 3.1, the same for all lines (page 15 of Avrett \& Loeser 2008). Both techniques still err in assuming SB opacities for the Kurucz lines, contrary to the NLTE overionization affecting e.g., all Fe I lines. The most detailed inclusion of these line-haze lines through proper multi-level NLTE synthesis is in Fontenla et al. (2015).

So far, best-fit codes (incorrectly called inversion codes) that iteratively adjust datafitting atmosphere stratifications for lines such as Fe I 6301.5 ^ ignore opacity NLTE from ultraviolet bf depletion and the accompanying line haze issue. They represent automation of the best-fit modeling by Holweger (1967) which led to the much-used model of Holweger \& Müller (1974). It ignored ultraviolet opacity depletion (Rutten \& Kostik 1982) and, worse for abundance determination, granulation (Asplund et al. 2004). While complete NLTE line-haze synthesis remains too demanding in data-fit iteration, recipes as the one defined in Bruls et al. (1992) and available in RH may serve as shortcut estimator.

Ly $\alpha$ within $A L C 7$. Ly $\alpha$ is the strongest and most scattering line in the solar spectrum. Figure 5 shows its properties across the ALC7 chromosphere. Its scattering decline occurs higher up, in the transition region out of ALMA reach. The value of $\varepsilon$ is exceedingly small: 

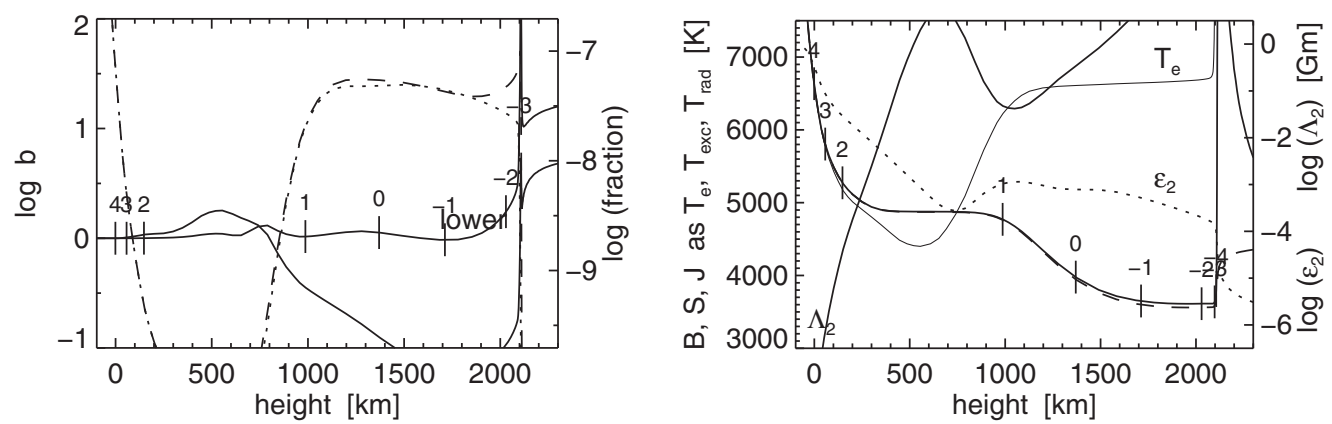

Figure 6. $\mathrm{H} \alpha$ in and from the ALC7 chromosphere, in the format of Fig. 3.

Ly $\alpha$ photons are typically scattered a million times before getting absorbed. Nevertheless, because the extinction coefficient is enormous (Fig. 1) the Doppler-core thermalization length in geometrical units $\sqrt{\pi} / \alpha \varepsilon$ is also small: only of order $10^{-5} \mathrm{Gm}=10 \mathrm{~km}$ in the low ALC7 chromosphere. This is an underestimate because so deep in the atmosphere $\left(\tau \approx 10^{8}\right.$ at line center!) the extended damping wings (from linear Stark broadening with the Holtsmark distribution) become important and provide longer scattering paths requiring $\mathrm{PRD}$ evaluation. Below I estimate them to reach up to a few hundred $\mathrm{km}$.

Even while scattering tremendously the $\operatorname{Ly} \alpha$ radiation remains well contained locally within the ALC7 chromosphere. It is boxed-in well enough that below the ALC7 transition region detailed radiative balance is a good approximation: just as many photons go up as go down, so that the net radiative rate is near zero (page 662 of Vernazza et al. 1981). This means that $\left(b_{l} / b_{u}\right) J \approx B$, which holds when $S \approx J$ without local radiative cooling or heating contribution $\alpha(J-S)$ (page 49 of RTSA).

Additionally, the short thermalization lengths thanks to the large extinction cause also local collisional balancing in the Ly $\alpha$ jump setting $J \approx B, b_{2} \approx b_{1}$ and $S \approx B$ up to large height (Eq. 3.41 on page 51 of RTSA). Since $b_{1} \approx 1$ because the ground level contains almost all hydrogen also $b_{2} \approx 1$, as demonstrated in the lefthand panel of Fig. 5 .

The LTE fractional population curve (left, dotted) shows a downturn from unity produced by increasing SB ionization, but this is compensated by a rise in $b_{l}$ which results from the onset of Ly $\alpha$ photon losses towards the transition region, especially in the inner wings as shown by the multiple source functions at right. These have corresponding $b_{2}<b_{1}$ divergence, but the $b_{1}$ rise compensates so that $b_{2} \approx 1$ to large height.

Thus, the most scattering line in the solar spectrum has near-LTE extinction and a near-LTE source function in the ALC7 chromosphere. The reasons are that the opacity in Ly $\alpha$ is enormous from the hydrogen abundance and from being the ground-level resonance line without much ionization, and that detour paths via other hydrogen transitions do not count. Ly $\alpha$ is the quintessential two-level scattering line, scatters as it likes in detailed radiative balance, and so dictates the $n_{2}$ population governing other hydrogen rates. The result is that within the ALC7 chromosphere Ly $\alpha$ is an LTE agent in defining $\mathrm{H} \alpha$ extinction and constraining mm extinction as discussed below. In the actual solar chromosphere it is also the major agent for these but in non-E fashion (below).

$H \alpha$ from $A L C 7$. Figure 6 shows that $\mathrm{H} \alpha$ escapes from the middle ALC7 chromosphere. Its extinction obeys the SB $\equiv$ LTE estimate closely, thanks to Ly $\alpha$. The fractional lowerlevel population therefore has a deep Boltzmann dip in the temperature minimum which implies that one cannot observe the upper photosphere in this line (Schoolman 1972). Indeed it primarily shows fibrils at line center and granules (and occasional Ellerman bursts) in its wings but no shock scenes as in Ca II H\& K (Rutten et al. 2008). 
Above the photosphere the thermalization length is above $10000 \mathrm{~km}$ so there is no chromospheric $S-B$ coupling. Like $\mathrm{Ly} \alpha, \mathrm{H} \alpha$ is pretty much its own boss in its scattering and so sets $b_{3}$ with respect to $b_{2}$. Ly $\beta$ shares $b_{3}$ and the $\mathrm{H} \alpha$ photon losses without affecting these because it remains in detailed radiative balance at these heights: when Ly $\beta$ photons get converted into escaping $\mathrm{H} \alpha$ photons plus Ly $\alpha$ photons then its $S \approx J$ diminishes accordingly (Fig. 2).

The $\mathrm{H} \alpha$ source function shows a normal scattering decline across the ALC7 chromosphere $\left(b_{u}<b_{l}\right.$ divergence at left) that is similar to that of Ca II $8542 \AA$ (display $15=$ Ca II $8542 \AA$ from ALC7), but it shows discordant behavior across the temperature minimum which is due to radiation build-up across this opacity chasm by back-scattering from the optically thick ALC7 chromosphere (Rutten \& Uitenbroek 2012). For H $\alpha$ the ALC7 chromosphere is simply an overlying scattering attenuator. Without it the scattering decline $S(\tau)$ would be the same but lie in the photosphere as in Fig. 3; indeed a radiative-equilibrium model without chromosphere has that and yet produces the same $\mathrm{H} \alpha$ profile ("Ha-Ha" comparison in Fig. 7 and Fig. 8 of Rutten \& Uitenbroek 2012).

Most $\mathrm{H} \alpha$ photons that escape near $1400 \mathrm{~km}$ from ALC7 were created at the bottom of the photosphere where $\varepsilon$ is large. They scatter out with increasing steps ( $\Lambda$ curve), then hit and scatter through the chromosphere until they escape around $\tau=1$. The actual large-contrast intensity pattern of the granulation imposed at the creation height gets erased by the subsequent scattering, so that lower-contrast chromospheric patterns (as fibrils) can yet show up, as beautifully demonstrated in Fig. 7 of Leenaarts et al. (2012) by comparing full 3D-scattering $\mathrm{H} \alpha$ synthesis with 1D column-wise $\mathrm{H} \alpha$ synthesis.

The older $\mathrm{H} \alpha$ literature following Thomas (1957) and Jefferies \& Thomas (1959) has called $\mathrm{H} \alpha$ photoelectrically-controlled, meaning domination of the $\eta$ detour terms in Eq. 3.1, but in the ALC7 and FCHHT-B atmospheres these contribute only $1 \%$ to $S-J$ in the temperature minimum and then rise to only about $5 \%$ across the chromosphere (Fig. 12 of Rutten \& Uitenbroek 2012), as part of the ionization circuit discussed below.

The upshot is that $\mathrm{H} \alpha$ is a rather ordinary scatterer, be it with special irradiation from below across its deep Boltzmann gap and with special SB opacity thanks to sitting on top of Ly $\alpha$. The emergent intensity $I \approx S(\tau=1)$ is lower for features with larger opacity (Schoolman 1972). In static models the detour contribution becomes dominant only in the transition region; in the actual Sun when and where $\mathrm{H} \alpha$ gets very bright from hydrogen recombination as in moss and flare rims in less quiescent conditions.

Hydrogen ionization in $A L C \%$. The $n_{2}$ population is fixed by Ly $\alpha$ through detailed radiative balancing and tight enclosure. The top of the hydrogen atom from $n=2$ at $10.2 \mathrm{eV}$ to the continuum at $13.6 \mathrm{eV}$ may be regarded as an extreme alkali-like atom with only $3.4 \mathrm{eV}$ ionization energy in which the Balmer lines act as quasi-resonance lines and the quasi-ground-level population $n_{2}$ is set by Ly $\alpha$. The main processes are photo-ionization in the Balmer and higher continua and cascade recombination along high levels.

In SE a closed hydrogen-top circuit operates in which NLTE photo-ionization driving by $T_{\mathrm{BaC}}^{\mathrm{rad}} \approx 5300 \mathrm{~K} \neq T_{\mathrm{e}}$ (bottom panels of Fig. 2 ) is balanced by cascade return along levels $i>4$ (page 663 of Vernazza et al. 1981 but beware of the Menzel/Zwaan flip). The resulting $b_{\text {cont }}$ curves represent additions to the Ly $\alpha$-set $b_{2}$ curves, positive in the temperature minimum where $T<5300 \mathrm{~K}$ and negative in the chromosphere where $T>$ $5300 \mathrm{~K}$, as illustrated by its reverse $b_{2} / b_{\text {cont }}$ in Fig. 4 .

Although the large chromospheric $\mathrm{H} \alpha S / B \approx b_{3} / b_{2}$ divergence is set by scattering photon losses in this line, these are not an important driver in this circuit because their loss does not increase $n_{2}$ as in alkali photon suction, due to the Ly $\alpha$-enforced $b_{2} \approx b_{1}$ 
equilibrium with $n_{1}$ which is a much larger electron reservoir than the continuum. Let me clarify: when a Na I $D_{1}$ photon leaves the Sun this enhances the Na I photo-ionization rate by putting an extra electron in the $\mathrm{NaI}$ ground level. The Na I line photon losses are a driver in establishing an ionization/recombination replenishment circuit in SE. However, when an $\mathrm{H} \alpha$ photon leaves the Sun it leaves an electron in level 2 that jumps down and back up a million or so times in Ly $\alpha$ before ending up in level 1 through collisional deexcitation, unnoticeably enhancing the temperature and the gigantic level-1 population. This way $\mathrm{H} \alpha$ is unusual in not increasing its lower-level $b$ with its photon losses as other strong lines do (e.g., display $15=$ Ca II $8542 \AA$ from ALC7 and display 16 $=\mathrm{Ca}$ II $\mathrm{K}$ from ALC7) - but those do not ride on Ly $\alpha$.

Overionization in the Balmer continuum is therefore the main hydrogen-top driver. The cascade recombination back to $n=2$ preferentially follows $\Delta n=-1$ steps downward, ending with $\mathrm{H} \alpha$ and making that the main circuit-flow balancer to obtain SE. Chromospheric radiative $J<S$ cooling in $\mathrm{H} \alpha$ therefore roughly compensates radiative $J>S$ heating in the Balmer continuum; both are much larger than such contributions by other hydrogen transitions (Fig. 48 on page 68 of Vernazza et al. 1981, reproduced as Fig. 8.12 on page 187 of RTSA).

In the upper photosphere the hydrogen-top ionization-recombination circuit produces emission in infrared H I Rydberg lines (Carlsson \& Rutten 1992) similarly to the Mg I 12-micron emission lines (Carlsson et al. 1992; explanation in Rutten \& Carlsson 1994).

Hydrogen in $A L C 7$ and FCHHT-B. I now return to comparing hydrogen properties between these models using Fig. 2. In FCHHT-B the $S \approx J$ curves for Ly $\alpha$ and Ly $\beta$ have extended tails below the steep onsets to the chromosphere and the transition region that are not present in ALC7. They result from scattering over larger extent than would box-in the Lyman-line radiation sufficiently to follow these abrupt temperature changes. In the tails detailed radiative Lyman balancing still holds closely $\left(S \approx J \approx\left(b_{u} / b_{l}\right) B\right)$ but detailed collisional balancing does not hold $\left(b_{u} \neq b_{l}\right)$.

The $S \approx J$ tails across the FCHHT-B temperature minimum come from Ly $\alpha$ and Ly $\beta$ photons from the relatively hot chromosphere penetrating a few hundred $\mathrm{km}$ below it. The $S \approx J$ tails below the transition region penetrate over similar extent. The transition region in ALC7 is as steep, but occurs at lower density and has less effect. The Ly $\alpha$ tails cause increases of $b_{2}$ and increase $\mathrm{H} \alpha$ extinction substantially.

$\mathrm{H} \alpha$ behaves similarly in the two models apart from these boosts. The larger optical thickness of the ALC7 chromosphere from higher temperature and therefore Boltzmann population results in higher $J$ in the gap. FCHHT-B has larger $\mathrm{H} \alpha$ opacity above $1500 \mathrm{~km}$ but less lower down (fractional population curves), resulting in slower $\tau$ buildup and wider $\mathrm{H} \alpha$ core formation.

Ly $\beta$ shares $b_{3}$ with $\mathrm{H} \alpha$ so where $b_{2} \approx b_{1}$ they have the same $S \approx\left(b_{u} / b_{l}\right) B$ behavior, but the temperature representation separates their $S \approx J$ curves in the bottom panels, with given $b_{l} / b_{u}$ giving larger $T_{\mathrm{e}} / T_{\mathrm{exc}}$ at longer wavelength.

The Balmer edge behaves the same in the two models because it is is entirely defined by escape in the deep photosphere (Fig. 4).

\section{Hydrogen in dynamic non-E solar simulations}

Non-E stands for non-LTE plus non-SE, i.e., time-dependent populations so that the rate equations do not sum to zero as in Eq. 2.100 on page 32 of RTSA. For hydrogen it is usually called non-equilibrium ionization, but a better name is non-equilibrium Lyman balancing because the large size $(10.2 \mathrm{eV})$ of the Ly $\alpha$ jump is the main culprit. 
It inhibits fast settling into detailed collisional Ly $\alpha$ balancing at low temperature. The other hydrogen populations including $n_{\text {cont }}$ follow suit.

The reason is the Boltzmann factor in $C_{u l} / C_{l u}=\left(g_{l} / g_{u}\right) \exp \left(E_{u l} / k T\right)$ (Eq. 2.61 on page 23 of RTSA). The downward collision rate $n_{u} C_{u l}$ barely depends on temperature since any collider will do, regardless of speed. Therefore, the upward Ly $\alpha$ collision rate $n_{1} C_{12}$ shares the very steep Boltzmann increase shown by the $\mathrm{H} \alpha$ curve in Fig. 1 . Both rates scale with the electron density (Eqs. 3.32-3.33 on page 50 of RTSA).

The pertinent non-E publications describe simulations with the 1D HD code RADYN (Carlsson \& Stein 2002), the 2D MHD code Stagger (Leenaarts et al. 2007), and the 3D MHD code Bifrost (Leenaarts et al. 2012; Loukitcheva et al. 2015; Carlsson et al. 2016). In each simulation ubiquitous shocks occur that reach temperatures around $7000 \mathrm{~K}$ at electron densities around $10^{11} \mathrm{~cm}^{-3}$. The RADYN simulation showed that at chromospheric heights the Lyman lines remain close to detailed radiative balance in and after these shocks; this condition was then set as tractability assumption in the more demanding multi-D simulations.

Shocks are hot and dense and also have increased $N_{\mathrm{e}} / N_{\mathrm{H}}$ from partial hydrogen ionization. Hence in shocks $C_{12}$ is large and collisional Ly $\alpha$ balancing is fast, reaching equilibrium within seconds. On the contrary, in shock aftermaths in which the gas cools to low temperature and all hydrogen recombines, the settling time scale becomes very long, $10^{3}$ seconds or more (Fig. 6 of Carlsson \& Stein 2002).

In the shocks Ly $\alpha$ reaches $S \approx B$ just as within the ALC7 chromosphere (which has similar temperature and electron density). This was demonstrated in the bottom panels of Fig. 2 (movie version) of Leenaarts et al. (2007) where the non-E $n_{2}$ curves equal the LTE curves momentarily in shocks. However, the same figure shows that in the cooling post-shock aftermaths the hydrogen $n_{2}$ population hangs at the large LTE shock value until the next shock arrives, typically 3-5 minutes later, whereas the LTE prediction follows the actual temperature instantaneously and drops dramatically following the Boltzmann slopes in Fig. 1. The last panel of Fig. 1 (movie version) of Leenaarts et al. (2007) shows that $b_{2}$ can so reach values above 12 dex! While these are called NLTE overpopulations they actually are LTE underpopulations because that assumption is the bad one.

The hydrogen ionization is again set by balancing Balmer photo-ionization and cascade recombination (Fig. 3 of Carlsson \& Stein 2002). This hydrogen-top loop balances fast and again adds NLTE-SE $b_{\text {cont }}$ additions to the $b_{2}$ value controlled by Ly $\alpha$, positive or negative for temperature below or above the Balmer continuum radiation temperature of $5300 \mathrm{~K}$. This is illustrated in the second-to-last panel of Fig. 1 of Leenaarts et al. (2007) by the wider span of the $b_{\text {cont }}$ color scale, reaching above 15 dex in $2400 \mathrm{~K}$ aftermath clouds (the lower limit imposed in the simulation, cf. Leenaarts et al. 2011) in which the Balmer-continuum driving ratio $B_{\mathrm{BaC}}(5300 \mathrm{~K}) / B_{\mathrm{BaC}}(2400 \mathrm{~K})$ is 4 dex.

\section{Hydrogen in solar contrails}

My premise in Pub 1 is that many if not most long $\mathrm{H} \alpha$ fibrils represent contrails of cooling gas in which hydrogen recombines, each outlining the track of a small fast propagating heating event in which hydrogen got ionized that passed minutes before, as in the example of Rutten \& Rouppe van der Voort (2017).

This suggestion implies sudden precursor heating, as in the simulation shocks but to yet higher temperature and electron density and therefore with faster Ly $\alpha$ balancing giving SB extinction to $\mathrm{H} \alpha$ that becomes very large above $8000 \mathrm{~K}$ (Fig. 1). In the subsequent cooling and recombining aftermath the $\operatorname{Ly} \alpha$ balancing becomes slow at low temperature, 
maintaining the large initial $\mathrm{H} \alpha$ extinction at growing $b_{2}$ overpopulation while $n_{2}$ hangs at its high earlier SB value, instead of instantaneously following the diminishing temperature along the steep Boltzmann slopes in Fig. 1 down to very small extinction. I call this large non-E post-hot extinction "post-Saha-Boltzmann-extinction" (PSBE) and suggest that it furnishes $\mathrm{H} \alpha$ contrail visibility enriching observed solar $\mathrm{H} \alpha$ scenes with long fibrils.

For the H Iff mm continua governing solar ALMA images the instantaneous SE ionization/recombination loop in the hydrogen top causes a tilt-like modification of the steep $\mathrm{H} \alpha$ Boltzmann slopes in Fig. 1, with the Balmer continuum radiation temperature of $5300 \mathrm{~K}$ as pivot: diminishing the extinction from the SB value for $T>5300 \mathrm{~K}$ and increasing it for $T<5300 \mathrm{~K}$. However, such tilt corrections amount to only a few dex, minor compared with the 10-dex Boltzmann increase. At near-complete hydrogen ionization the $\mathrm{HI}$ If mm extinction anyhow remains as large as shown in Fig. 1, at longer wavelengths very much larger than the $\mathrm{H} \alpha$ extinction. Therefore I predict large mm extinction, hence ALMA visibility, for any feature with PSBE contrail visibility in $\mathrm{H} \alpha$.

Lateral contrail contrasts then depend on contrail histories. ALMA observes the current temperature from PSBE-boosted optically thick features, but for $\mathrm{H} \alpha$ the history not only boosts the feature opacity but larger opacity also darkens the line core along the scattering decline. $\mathrm{H} \alpha$ fibrils may so differ in darkness at similar temperature while the same fibrils seen with ALMA do not. Also, unlike $\mathrm{H} \alpha$ ALMA does not sense difference in Dopplershift. I therefore expect contrails to appear less fibrilar and more blanket-like in ALMA images.

The Ly $\alpha$ back-radiation tails for FCHHT-B in Fig. 2 suggest that Ly $\alpha$ scattering from precursors and contrails into cool surroundings produces aureole visibility by increasing the $n_{2}$ population and hence the HIff mm extinction.

\section{Discussion}

Summary. Chromospheric hydrogen diagnostics are dominated by the $10.2 \mathrm{eV}$ Ly $\alpha$ jump. Its large size causes very steep initial temperature sensitivity for $\mathrm{H} \alpha$ and $\mathrm{H} \mathrm{Iff}$ extinction (Fig. 1) and long recombination retardation during post-ionization cooling which implies large-extinction aftermaths of heating events in $\mathrm{H} \alpha$ and ALMA images.

Precursors. The nature of the proposed heating events producing long $\mathrm{H} \alpha$ fibrils as PSBE aftermath contrails is not known. The precursor in the example of Rutten \& Rouppe van der Voort (2017) appeared rather like spicules-II of which the agent is also unknown (Pereira et al. 2012), but with more horizontal launching. So far spicules-II and long $\mathrm{H} \alpha$ fibrils lack in Bifrost simulations and therefore also in the ALMA predictions of Loukitcheva et al. (2015). Bipolar ion-neutral separation may play a role where hydrogen ionizes (Martínez-Sykora et al. 2016). Small-scale torsion kicking may deliver long fibril extent because torsion waves are incompressive, travel far before dissipation, and appear ubiquitously (De Pontieu et al. 2014).

ALMA may be the best facility to study contrail precursors thanks to their large H Iff extinction. When ALMA reaches sufficient angular resolution it may faithfully map precursor morphology since free-free continua do not suffer scattering. It may also map cooler precursor and contrail aureoles that gain opacity from Ly $\alpha$ surround scattering.

Rydberg lines. A final solar-ALMA item (not in Pub 1 as too speculative for prediction) concerns high- $n$ H I lines. I suggest that some may be detectable with ALMA.

In the infrared the Mg I 7-6 lines at 12.2 and 12.3 micron due to ladder-wise cascade recombination as a replenishment flow (explanation in Rutten \& Carlsson 1994) are more 
conspicuous than the similarly formed H I 7-6 line at 12.4 micron because the $\operatorname{Mg}$ I $n=6$ levels have larger population in the upper photosphere (Fig. 15 of Carlsson et al. 1992). Towards higher $n$ the different Rydberg wavelengths converge to the hydrogenic limit so that higher- $n$ lines become blended mixtures of different species.

The highest $n$ levels, up to the formal $n=\infty$, vanish into the continuum due to collisional ionization-limit lowering; the limit for existence depends on density (pages 69 and 275 of Mihalas 1970). However, the EK results in Figs. 4 and 5 of Kunc \& Soon (1992) suggest that Rydberg levels with $\alpha(\Delta n=1)$ lines between them in the ALMA range may exist in chromospheric conditions.

Figure 3 of Carlsson \& Rutten (1992) shows predicted emission peak strengths $I / I_{\text {cont }}$ for the HI $\alpha$ lines with lower levels 4 to 18 for the plane-parallel SE model of Maltby et al. (1986) and suggests that these become negligible at higher $n$. The highest detected so far are 19- $\alpha$ (Clark et al. 2000a) and 21- $\alpha$ (Clark et al. 2000b), but only at the limb and fitting the predicted weakening (Fig. 6 of Clark et al. 2000a).

However, my proposed mechanisms for long $\mathrm{H} \alpha$ fibrils give all hydrogen-top levels much larger population than static-atmosphere SE modeling predicts. If they are not swamped by the also boosted HIff background HI Rydberg lines may be detectable in ALMA spectra and be freed of non-boosted blends. They may then appear as small and fast spectral emission features on the disk arising from heating events with steep temperature gradients and resulting $T\left(\tau_{\mu}=1\right)$ contrasts, and as coarser spectral-extent features at the limb due to PSBE-boosted cooling contrails with less temperature contrast but $\tau T$ visibility against the dark-sky background.

In the coming solar observing during ALMA Cycle 4 the H I 30- $\alpha$ line at $231.901 \mathrm{GHz}$ sits near the center of the 2-GHz wide sampling interval around $232 \mathrm{GHz}$ of Band 6 in which 0.5 arcsec resolution can be reached with the $538.9 \mathrm{~m}$ baseline. This represents the best candidate. In addition, $39-\alpha$ at $106.737 \mathrm{GHz}$ and $41-\alpha$ at $92.034 \mathrm{GHz}$ fall possibly just inside such sampling intervals at 105 and $93 \mathrm{GHz}$ in Band 3 (Kobelski \& ALMA Solar Development Team 2016; Rydberg frequencies from the NIST calculator).

If such lines are detected then measuring their Zeeman broadening or merging (Greve 1975; cf. Greve \& Pauls 1980) or even partial splitting as in Fig. 9 of Rutten \& Carlsson (1994) is an exciting prospect for magnetometry of the non-E chromosphere.

\section{Acknowledgements}

I thank S. Toriumi for inviting me to the National Astronomical Observatory of Japan where I made most of the above figures, the organizers of IAU Symposium 327 for a worthwhile and pleasant conference, and the Leids Kerkhoven-Bosscha Fonds for travel support.

\section{References}

Asensio Ramos, A., Trujillo Bueno, J., Carlsson, M., \& Cernicharo, J. 2003, ApJL, 588, L61 ADS

Asplund, M., Grevesse, N., Sauval, A. J., Allende Prieto, C., \& Kiselman, D. 2004, A\&A, 417, 751 ADS

Avrett, E. H. 1965, SAO Special Report, 174, 101 ADS

Avrett, E. H. \& Loeser, R. 2008, ApJS, 175, 229 ADS

Bruls, J. H. M. J., Rutten, R. J., \& Shchukina, N. G. 1992, A\&A, 265, 237 ADS

Carlsson, M., Hansteen, V. H., Gudiksen, B. V., Leenaarts, J., \& De Pontieu, B. 2016, A\&A, 585, A4 ADS

Carlsson, M. \& Rutten, R. J. 1992, A\&A, 259, L53 ADS

Carlsson, M., Rutten, R. J., \& Shchukina, N. G. 1992, A\&A, 253, 567 ADS 
Carlsson, M. \& Stein, R. F. 1995, ApJL, 440, L29 ADS

Carlsson, M. \& Stein, R. F. 2002, ApJ, 572, 626 ADS

Clark, T. A., Naylor, D. A., \& Davis, G. R. 2000a, A\&A, 357, 757 ADS

Clark, T. A., Naylor, D. A., \& Davis, G. R. 2000b, A\&A, 361, L60 ADS

De Pontieu, B., Rouppe van der Voort, L., McIntosh, S. W., et al. 2014, Science, 346, 1255732 ADS

Ellerman, F. 1917, ApJ, 46, 298 ADS

Fontenla, J. M., Avrett, E. H., \& Loeser, R. 1993, ApJ, 406, 319 ADS

Fontenla, J. M., Curdt, W., Haberreiter, M., Harder, J., \& Tian, H. 2009, ApJ, 707, 482 ADS

Fontenla, J. M., Stancil, P. C., \& Landi, E. 2015, ApJ, 809, 157 ADS

Greve, A. 1975, SoPh, 44, 371 ADS

Greve, A. \& Pauls, T. 1980, A\&A, 82, 388 ADS

Greve, A. \& Zwaan, C. 1980, A\&A, 90, 239 ADS

Holweger, H. 1967, Z. Astrophys., 65, 365 ADS

Holweger, H. \& Müller, E. A. 1974, SoPh, 39, 19 ADS

Jefferies, J. T. 1968, Spectral line formation ADS

Jefferies, J. T. \& Thomas, R. N. 1959, ApJ, 129, 401 ADS

Kobelski, A. \& ALMA Solar Development Team. 2016, in Astron. Soc. Pacific Conf. Series, Vol. 504, Coimbra Solar Physics Meeting: Ground-based Solar Observations in the Space Instrumentation Era, ed. I. Dorotovic, C. E. Fischer, \& M. Temmer, 327 ADS

Kunc, J. A. \& Soon, W. H. 1992, ApJ, 396, 364 ADS

Kurucz, R. L. 2009, in Am. Inst. Phys. Conf. Series, ed. I. Hubený, J. M. Stone, K. MacGregor, \& K. Werner, Vol. 1171, 43-51 ADS

Leenaarts, J., Carlsson, M., Hansteen, V., \& Gudiksen, B. V. 2011, A\&A, 530, A124 ADS

Leenaarts, J., Carlsson, M., Hansteen, V., \& Rutten, R. J. 2007, A\&A, 473, 625 ADS

Leenaarts, J., Carlsson, M., \& Rouppe van der Voort, L. 2012, ApJ, 749, 136 ADS

Loukitcheva, M., Solanki, S. K., Carlsson, M., \& White, S. M. 2015, A\&A, 575, A15 ADS

Maltby, P., Avrett, E. H., Carlsson, M., et al. 1986, ApJ, 306, 284 ADS

Martínez-Sykora, J., De Pontieu, B., Carlsson, M., \& Hansteen, V. 2016, ApJL, 831, L1 ADS

Mihalas, D. 1970, Stellar atmospheres ADS

Pereira, T. M. D., De Pontieu, B., \& Carlsson, M. 2012, ApJ, 759, 18 ADS

Rutten, R. J. 1988, in Astrophys. Space Sci. Library, Vol. 138, IAU Colloq. 94: Physics of Formation of Fe II Lines Outside LTE, ed. R. Viotti, A. Vittone, \& M. Friedjung, 185 ADS

Rutten, R. J. 2002, Journal of Astronomical Data, 8 ADS

Rutten, R. J. 2003, Radiative Transfer in Stellar Atmospheres ADS

Rutten, R. J. 2017, A\&A, 598, A89 (ADS code 2017A\&A...598A..89R).

Rutten, R. J. 2016b, A\&A, 590, A124 ADS

Rutten, R. J. \& Carlsson, M. 1994, in IAU Symposium, Vol. 154, Infrared Solar Physics, ed. D. M. Rabin, J. T. Jefferies, \& C. Lindsey, 309 ADS

Rutten, R. J. \& Kostik, R. I. 1982, A\&A, 115, 104 ADS

Rutten, R. J. \& Rouppe van der Voort, L. H. M. 2017, A\&A, 597, A138 (ADS code 2017A\&A...597A.138R).

Rutten, R. J. \& Uitenbroek, H. 2012, A\&A, 540, A86 ADS

Rutten, R. J., van Veelen, B., \& Sütterlin, P. 2008, SoPh, 251, 533 ADS

Rutten, R. J., Vissers, G. J. M., Rouppe van der Voort, L. H. M., Sütterlin, P., \& Vitas, N. 2013, J. Phys. Conf. Ser., 440, 012007 ADS

Schoolman, S. A. 1972, SoPh, 22, 344 ADS

Spruit, H. C. 1977, PhD thesis, Thesis University of Utrecht, The Netherlands. ADS

Thomas, R. N. 1957, ApJ, 125, 260 ADS

Uitenbroek, H. 2000, ApJ, 536, 481 ADS

Uitenbroek, H. 2001, ApJ, 557, 389 ADS

Uitenbroek, H. \& Bruls, J. H. M. J. 1992, A\&A, 265, 268 ADS

Vernazza, J. E., Avrett, E. H., \& Loeser, R. 1981, ApJS, 45, 635 ADS

Vitas, N., Viticchiè, B., Rutten, R. J., \& Vögler, A. 2009, A\&A, 499, 301 ADS

Wedemeyer, S. 2016, The Messenger, 163, 15 ADS 